\title{
Incidence of Serous Tubal Intraepithelial Carcinoma (STIC) by Algorithm Classification in Serous Ovarian Tumor Associated with PAX8 Expression in Tubal Epithelia: A Study of Single Institution in Japan
}

\author{
Satoru Munakata, M.D., Ph.D. and Toshiya Yamamoto, M.D., Ph.D.
}

\begin{abstract}
Summary: Serous ovarian carcinoma is now hypothesized to originate from fallopian tube epithelium (FTE). We investigated the FTE abnormalities in the patients with epithelial ovarian tumors. Our study included 55 cases of serous tumors ( 24 carcinomas, 8 borderline tumors, and 23 adenomas), 14 mucinous carcinomas, 22 endometrioid carcinomas, 5 clear cell carcinomas, and 2 malignant Brenner tumors. FTE was diagnosed by the diagnostic algorithm, which combines the data of morphology, and p53, Ki-67 immunostaining, as serous tubal intraepithelial carcinoma, serous tubal intraepithelial lesion, p53 signature, and normal/reactive. Serous tubal intraepithelial carcinoma, serous tubal intraepithelial lesion, p53 signature, and normal/reactive were observed in 5, 3, 0, and 16 cases in serous carcinoma; $0,3,0$, and 5 cases in serous borderline tumor; $0,1,1$, and 21 cases in serous adenoma; $0,0,1$, and 13 cases in mucinous carcinoma; $0,0,3$, and 19 cases in endometrioid carcinoma; 0, 0, 0, and 5 cases in clear cell carcinoma; and 0,1,0, and 1 case in malignant Brenner tumor. Among tumors of serous histology and between carcinomas, FTE abnormalities differed significantly $(P<0.05)$. Serous tubal intraepithelial carcinomas were only found in serous carcinoma. The incidence of secretory cell proliferation (SCP) was examined by PAX8 expression. The rate of SCP was extremely high in serous carcinoma $(96 \%)$. Among tumors of serous histology and between carcinomas, an incidence of SCP differed significantly $(P<0.05)$. Patients with SCP were significantly older $(P<0.0001)$. Our observations were concordant with the hypothesis of serous ovarian carcinogenesis. The SCP has a meaningful association with serous ovarian cancer. Key Words: Serous ovarian tumor-Serous tubal intraepithelial carcinoma-PAX8-Immunohistochemistry-Japanese institution.
\end{abstract}

From the Departments of Pathology (S. M.); and Obstetrics and Gynecology (T. Y.), Sakai City Hospital, Sakai, Osaka, Japan.

The authors declare no conflict of interest.

Address correspondence and reprint requests to Satoru Munakata, MD, PhD, Department of Pathology, Sakai City Hospital, 1-1-1 Minami-Yasui-Cho, Sakai-Ku, Sakai, Osaka 5900064, Japan. E-mail: munak-s@sakai-hospital.jp.

This is an open-access article distributed under the terms of the Creative Commons Attribution-NonCommercial-NoDerivitives 3.0 License, where it is permissible to download and share the work provided it is properly cited. The work cannot be changed in any way or used commercially.
Incidence of ovarian cancer has been very low in Japan compared with western countries, but it is increasing recently (1). Mortality rate of ovarian cancer is also rising. Therefore, ovarian cancer has been very important among gynecologic malignancies in Japan.

Recent morphologic, immunohistochemical, and molecular genetic studies have led to the development of a new paradigm for the pathogenesis and origin of 
epithelial ovarian cancer. A dualistic model of carcinogenesis divides epithelial ovarian cancer into 2 categories, Type I and Type II (2). Accordingly, serous ovarian cancers are divided into the 2 categories.

To reduce the lifetime-risk of hereditary ovarian cancer that is related to $B R C A 1$ or $B R C A 2$ gene mutation, prophylactic salpingo-oophorectomy is widely accepted in western countries (3-5). Thorough examination of these fallopian tubes revealed frequent association of serous tubal intraepithelial carcinoma (STIC) or tubal intraepithelial dysplasia, which usually has p53 mutations (6-13).

Studies to find STICs are most often based on the morphology by hematoxylin and eosin (H\&E) staining where diagnostic reproducibility is low (14). More recently, an algorithm for the diagnosis of STICs has been proposed with the use of $\mathrm{p} 53$ and $\mathrm{Ki}-67$ immunostaining $(15,16)$. Using the algorithm interobserver diagnostic agreement has been improved (16).

$P A X 8$ is a transcription factor that is essential for embryonic development of the kidney, müllerian organs, and thyroid. In tubal epithelial cells secretory cells and basal cells, but not ciliated cells, express PAX8 (17). PAX2 is a member of the paired box transcription factor that is critical in the embryogenesis and organogenesis of the central nervous system, eye, ear, and the genitourinary tract. PAX2 is expressed in the secretory cells of the fallopian tube epithelium (FTE). Nuclear immunolocalization parallels that of PAX8. Recently, PAX2-null secretory cell outgrowths of the fallopian tube were reported that they were frequently associated with serous borderline tumors (18). In contrast, PAX8 is always expressed in the nuclei of the tubal secretory cells. We defined the outgrowth of PAX8-positive tubal secretory cells as secretory cell proliferation (SCP).

In this study we investigated the incidence of STICs by using the diagnostic algorithm among serous tumors and between carcinomas of different types of histology to know the association of tubal epithelial abnormalities with epithelial ovarian tumor to testify the hypothesis, in which serous ovarian carcinoma originate from FTE. We also investigated the frequency of SCP by PAX8 expression.

\section{MATERIALS AND METHODS}

\section{Case Selection}

This study was approved by the institutional review board of Sakai City Hospital. Cases were defined as women with epithelial ovarian cancer, who underwent surgery between April 2003 and June 2013. Data for the cases were retrieved from the pathology database of Sakai City Hospital. Eighty cases were identified. The cases, whose fallopian tube could be examined histologically were selected for the study. Twelve cases were excluded because blocks of fallopian tube were not available for histologic evaluation. One case was not included because the case was tubal cancer. Sixty-seven cases were included in the study. For comparison, we also included the 8 cases with serous borderline tumor and 23 cases with serous adenoma. Clinical features of the cases were described in Table 1. In 24 cases of serous carcinoma, all cases except one were high grade.

\section{Immunohistochemistry}

One or 2 blocks containing ipsilateral or contralateral fallopian tube were chosen from each case. Only $10 \%(10 / 98)$ of the cases were cut extensively, by Sectioning and Extensively Examining the FIMbriated end (SEE-FIM) protocol (12). Additional 5 cases were prepared extensively, so $15 \%$ (15/98) were considered as more than optimal. In the fallopian tubes examined, 49 cases $(50 \%)$ included fallopian tube fimbriae. Paraffin sections of $3 \mu \mathrm{m}$ were cut and stained with $\mathrm{H} \& \mathrm{E}$ and immunohistochemistry using primary antibodies, including p53 (clone DO-7, ready-to-use; Nichirei, Tokyo, Japan), Ki-67 (clone MIB-1, 1:50; Dako, Tokyo, Japan), PAX8 (clone PAX8R1, 1:50; Santa Cruz, CA), and antibasal bodies of cilia LhS28 (clone LhS28, 1:1000; Abcam, Cambridge, UK). Heat-induced pretreatment was applied for p53, Ki-67, and LhS28. VENTANA BenchMark XT system was employed for immunohistochemistry.

\section{Histologic Evaluation and Application of the Diagnostic Algorithm}

Histologic features of each fallopian tube were morphologically evaluated as unequivocal for STIC (UES) (Figs. 1, 2), suspicious for STIC (SS), or not suspicious for STIC (NS) by diagnostic criteria, shown by Visvanathan et al. (15). They used diagnostic criteria for morphology, which defined normal/reactive as having $<2$ diagnostic features in any length of nonciliated cells, atypical as having 2 diagnostic features in 10 or more consecutive nonciliated cells, or having 2 or more features in $<10$ consecutive nonciliated cells, and STIC as having 2 or more features in 10 or more consecutive nonciliated cells. 
TABLE 1. Clinical features of the cases

\begin{tabular}{|c|c|c|c|c|c|c|}
\hline Histology & No. cases & Mean age (range) & Stage* I & Stage II & Stage III & Stage IV \\
\hline Serous tumor (total) & 56 & $55(23-86)$ & - & - & - & - \\
\hline Carcinoma & 24 & $61(35-86)$ & 3 & 1 & 19 & 1 \\
\hline Borderline tumor & 8 & $53(27-76)$ & - & - & - & - \\
\hline Adenoma & 23 & $57(23-79) \dagger$ & - & - & - & - \\
\hline Mucinous carcinoma & 14 & $61(20-77)$ & 11 & 0 & 3 & 0 \\
\hline Endometrioid carcinoma & 22 & $53(22-77)$ & 14 & 3 & 5 & 0 \\
\hline Clear cell carcinoma & 5 & $52(41-71)$ & 4 & 0 & 1 & 0 \\
\hline Malignant Brenner tumor & 2 & $64(61-67) \dagger$ & 2 & 0 & 0 & $0 \ddagger$ \\
\hline Total & 98 & $57(20-86)$ & 35 & 4 & 28 & 1 \\
\hline
\end{tabular}

*FIGO (surgical-clinical) staging.

$\uparrow$ Age distribution was not statistically significant between serous tumors and epithelial ovarian carcinomas.

† Stage distribution was statistically significant between epithelial ovarian carcinomas $(P<0.0001)$.

The diagnostic features included nuclear enlargement, marked pleomorphism, abnormal chromatin, mitotic figure, epithelial stratification, nuclear molding, and apoptotic bodies. Final diagnoses were based on the diagnostic algorithm with combination of morphologic diagnosis and immunohistochemical results shown by Visvanathan et al. (15) (Table 2). Immunohistochemical evaluation included p53 and Ki-67. p53 was positive for the cases with diffuse intense staining over $75 \%$ of cells or completely negative staining on
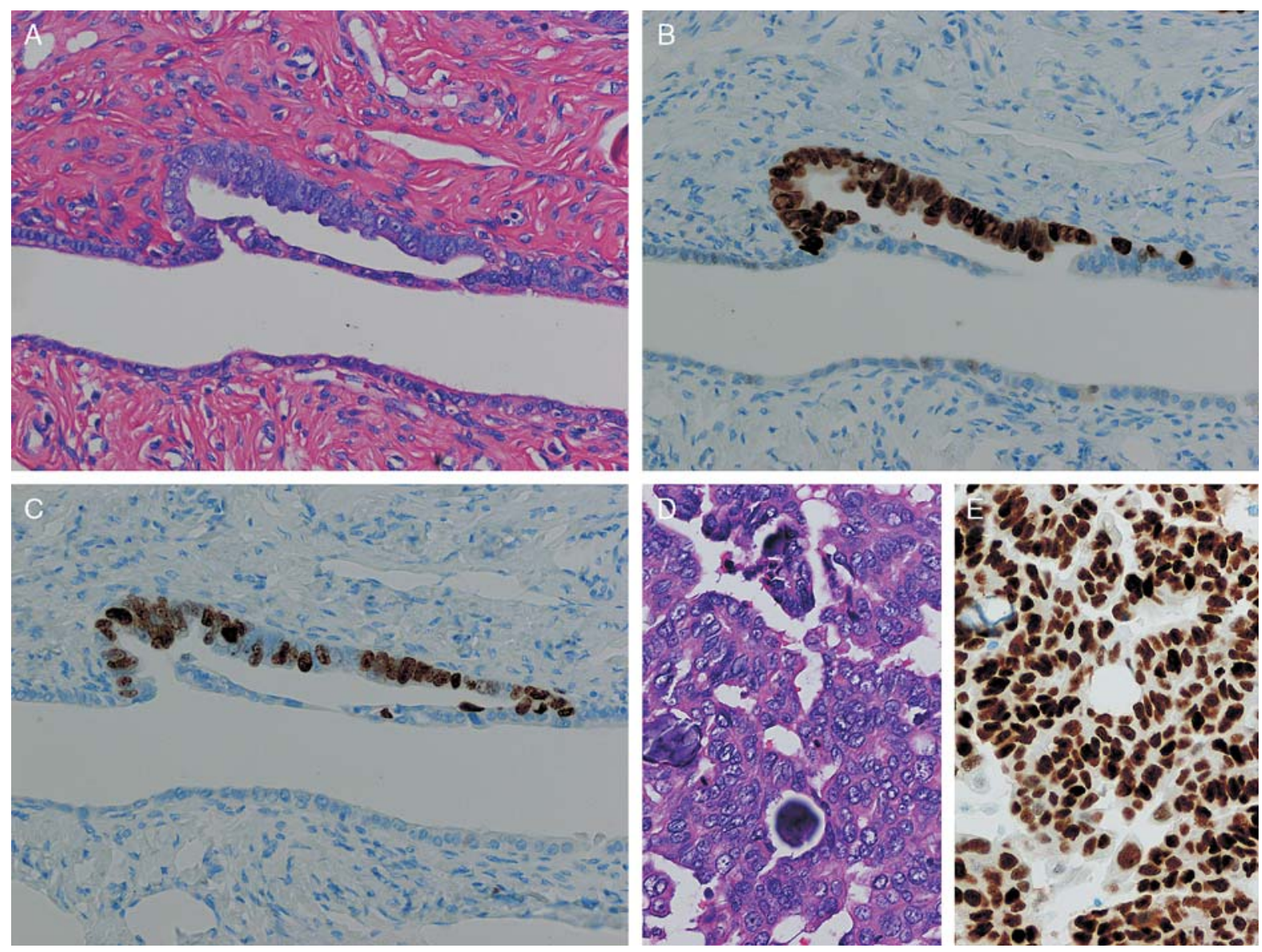

FIG. 1. (A) Unequivocal for serous tubal intraepithelial carcinoma by hematoxylin and eosin staining. Tubal epithelia showed nuclear atypia. (B) p53 was strongly expressed in the tubal epithelium. (C) Ki-67 was frequently positive in the epithelium (Ki-67 labeling index $53 \%$ ). (D) Morphology of the high-grade serous carcinoma of the same patient. Psammoma body is observed in the lower area. (E) p53 immunostaining on the same tumor. Strong nuclear staining is present [original magnification: $20 \times$ for $(\mathrm{A}-\mathrm{E})$ ]. 

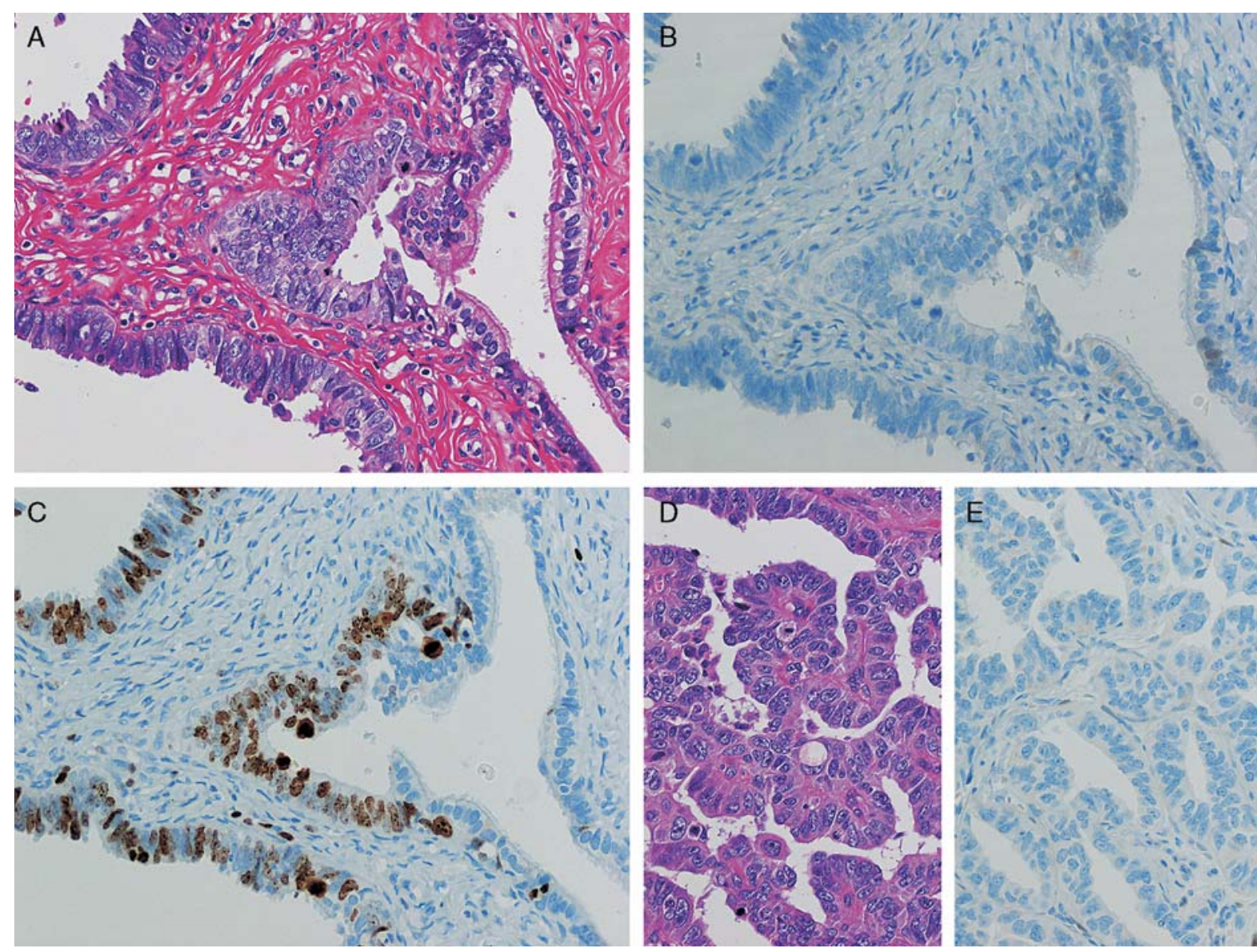

FIG. 2. (A) Unequivocal for serous tubal intraepithelial carcinoma by hematoxylin and eosin staining. Tubal epithelia showed nuclear atypia. (B) p53 was completely negative for the atypical epithelial cells. Note weakly positive staining in the normal epithelium in the right side of the image. (C) Ki-67 was frequently positive in the epithelium (Ki-67 labeling index 34\%). (D) Morphology of the high-grade serous carcinoma of the same patient. (E) p53 immunostaining on the same tumor. Completely negative staining is observed [original magnification: $20 \times$ for $(\mathrm{A}-\mathrm{E})$ ].

targeted area as well as the same staining pattern in tumor cells (Figs. 1, 2). Ki-67 labeling index $\geq 10 \%$ was designated as positive. The final diagnosis was based on a combination of the morphology-only diagnosis and coordinate immunohistochemical scores as per the algorithm (Table 2). Those included STIC (Figs. 1, 2), serous tubal intraepithelial lesion (STIL) (Fig. 3), p53 signature (Fig. 3), and normal/reactive.

\section{Evaluation of PAX8 and LhS28 Expression}

SCP was examined by PAX8 and LhS28. PAX8 was only stained in the nuclei of the tubal secretory cells, but not in the ciliated cells. LhS28 is an antibasal body marker of ciliated cells, so the staining was observed on the cytoplasmic band lying adjacent to the apical membrane and in the region of the ciliated bodies of the ciliated cells, but not on the secretory cells. PAX8 and LhS28 staining was mutually exclusive in the tubal epithelial cells. Using these antibodies, SCP was easily discerned. Nuclear staining of PAX8 in 2 or more sets of 10 or more consecutive tubal epithelial cells was considered as positive for SCP. If the area of PAX8-positive cells exceeded a half of the total epithelial area, the staining pattern was designated as PAX8 (E) (Figs. 4A, B). If the area of those clusters of PAX8positive cells was limited in less than half of the total epithelial cells, the staining pattern was designated as PAX8 (H) (Figs. 4D, E). Loss of ciliated cells was confirmed by loss of LhS28 expression (Figs. 4C, F).

\section{Statistics}

Comparison of patients' age between groups was made by 1-factor analysis of variance. Comparison of patients' age between groups of PAX8 expression was made by Student $t$ test. Comparison between clinical 
TABLE 2. Algorithm for the diagnosis of serous tubal intraepithelial carcinoma

\begin{tabular}{lll}
\hline Morphology & Immunohistochemistry & Final diagnosis \\
\hline Unequivocal for STIC & p53(+), Ki-67: high & STIC \\
& p53(+), Ki-67: low & STIL \\
& p53(-), Ki-67: high & STIL \\
puspicious for STIC & p53(-), Ki-67: low & STIL \\
& p53(+), Ki-67: high & STIC \\
& p53(-), Ki-67: high & STIL \\
p55IL \\
p53(-), Ki-67: low & Normal/ \\
Not suspicious for STIC & p53(+), Ki-67: high & rTIL \\
& p53(+), Ki-67: low & p53 signature \\
& p53(-), Ki-67: high & Normal/ \\
& p53(-), Ki-67: low & reactive \\
& & Normal/ \\
& & reactive \\
\hline
\end{tabular}

p53 is positive when the focus of cells with minimum of 12 cells showed $>75 \%$ cells with moderate to strong expression or $0 \%$ cells with expression as well as the tumor cells. Ki-67 is high when $\geq 10 \%$ cells showed staining, and low when $<10 \%$ cells showed staining.

STIC indicates serous tubal intraepithelial carcinoma; STIL, serous tubal intraepithelial lesion.

stages and histologic types was made by KruskalWallis 1-way analysis of variance. Correlation of malignant status and tubal epithelial abnormalities was calculated by Spearman rank correlation coefficient. Comparison between histologic types of epithelial cancer and tubal epithelial abnormalities was made by $\chi^{2}$ for independence test. Comparison between histologic types of epithelial cancer and PAX8 positivity was made by Mann-Whitney $U$ test. All of the statistics were made by using STATCEL software (OMS, Japan).

\section{RESULTS}

\section{Age and Clinical Stage}

Mean age of the patients and FIGO (surgical-clinical) stage of cancer in each histologic type were shown in Table 1. Mean ages of the patients with serous tumors were not statistically different between tumors with different malignant potential. Mean ages of the patients with cancer were not different between different histologic types. Cases in advanced FIGO stages (stages II-IV) with serous carcinoma, mucinous carcinoma, endometrioid carcinoma, clear cell carcinoma, and malignant Brenner tumor were $88 \%, 21 \%, 36 \%, 20 \%$, and $0 \%$, respectively. Stage distribution was significantly different between histologic types of the tumor $(P<0.0001)$.

\section{Tubal Epithelial Abnormalities by H\&E Staining}

Data for tubal epithelial abnormalities by H\&E staining are shown in Table 3. In serous adenocarcinoma, UES, SS, and NS evaluated by H\&E-stained
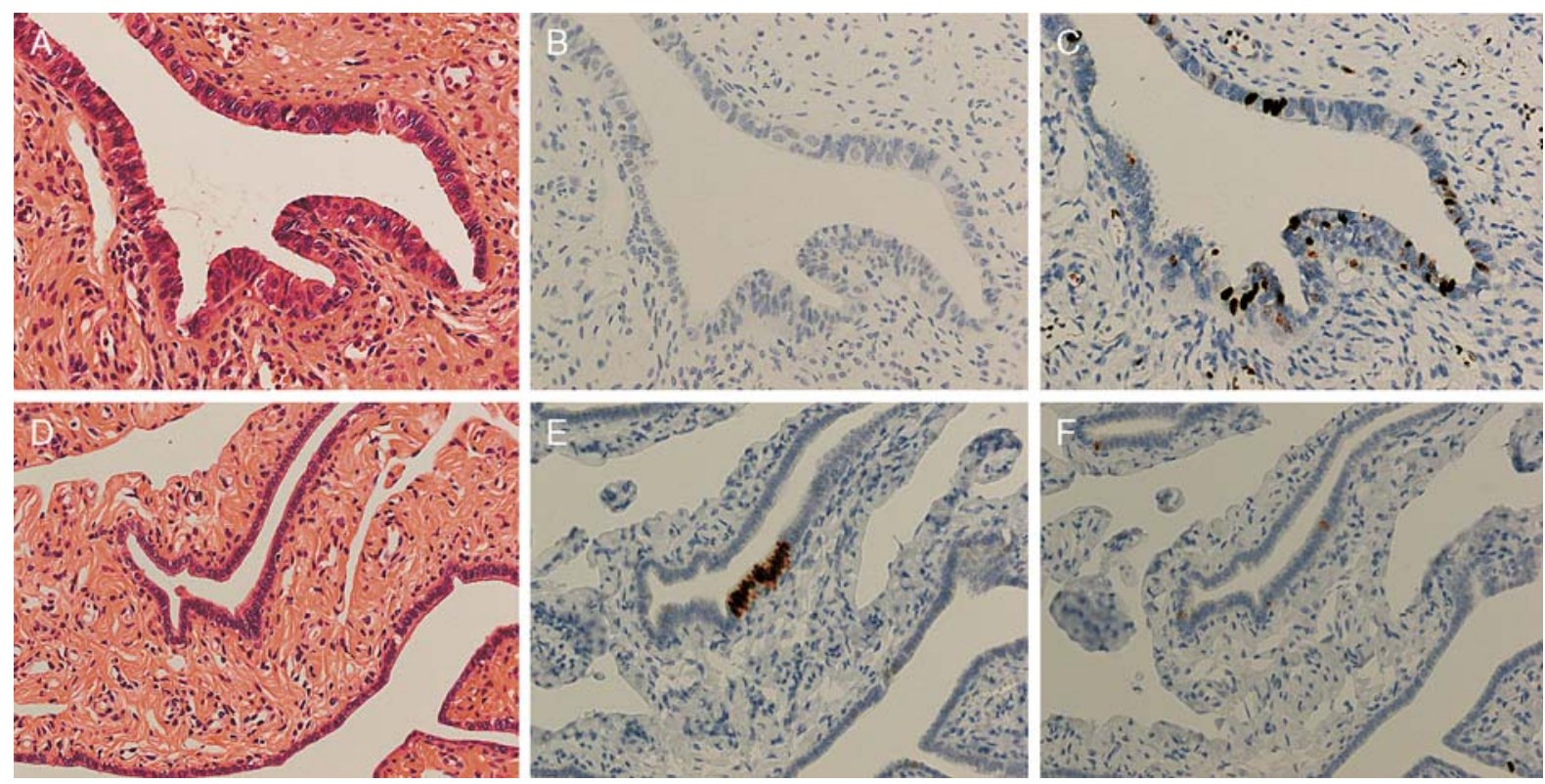

FIG. 3. (A) Suspicious for serous tubal intraepithelial carcinoma (STIC) by hematoxylin and eosin (H\&E) staining. Tubal epithelia showed nuclear enlargement and molding. (B) p53 was negative. (C) Ki-67 labeling index was $28 \%$ for the lesion. Tubal epithelia of this region were diagnosed as serous tubal intraepithelial lesion. (D) Not suspicious for STIC by H\&E staining. (E) p53 was positive in more than 12 consecutive cells. (F) Ki-67 labeling index was $<10 \%$. Tubal epithelia of this region were diagnosed as p53 signature [original magnification: $20 \times$ for $(\mathrm{A}-\mathrm{F})]$. 

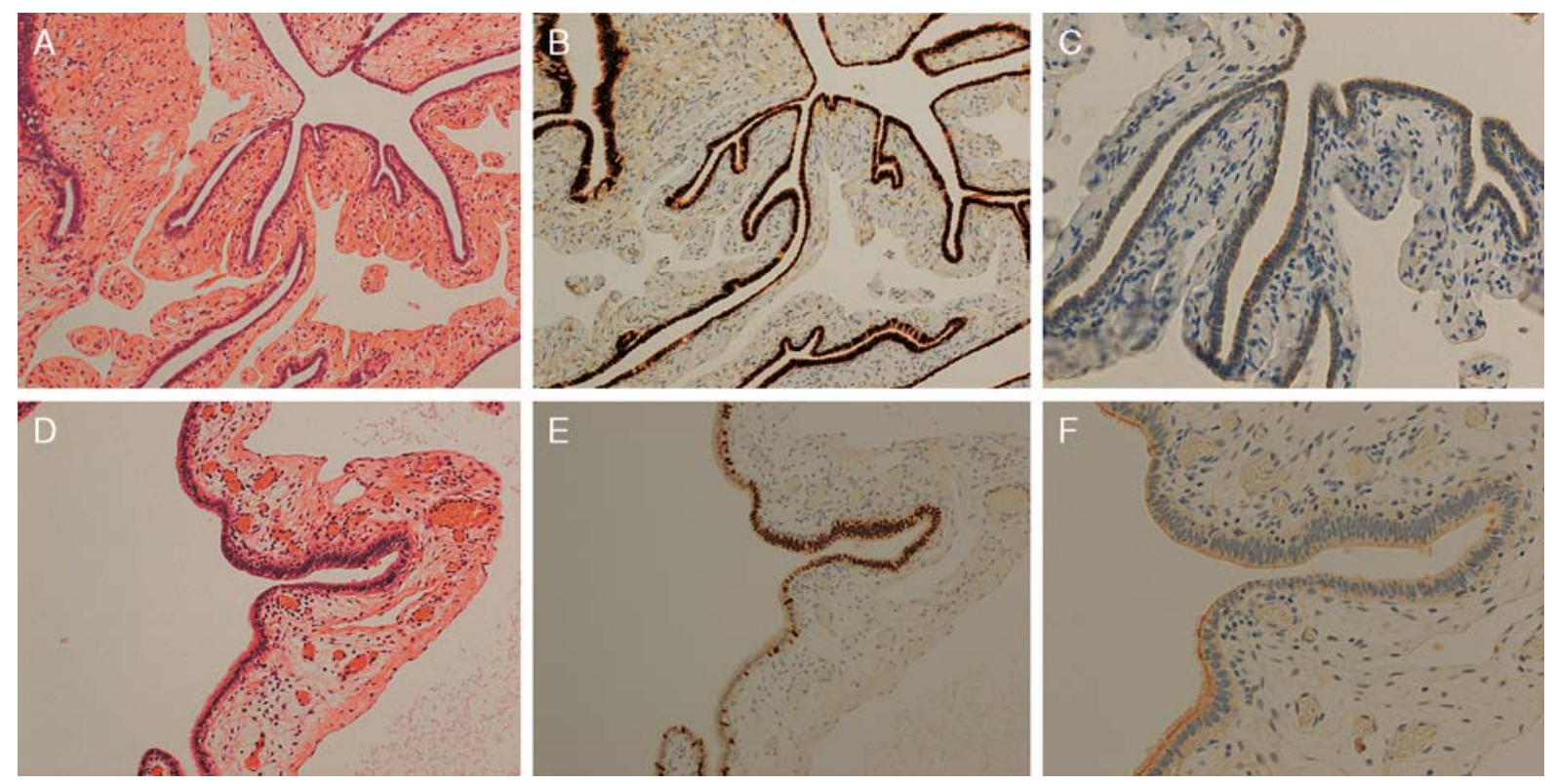

FIG. 4. (A) Morphology for fallopian tube with PAX8 (E). (B) Tubal epithelia with PAX8 (E) pattern showed diffuse staining of PAX8. (C) LhS28 staining revealed loss of cilia in the same epithelium. (D) Morphology for fallopian tube with PAX8 (H). (E) Tubal epithelial with PAX8 (H) pattern showed PAX8-positive staining in the center of the tubal epithelia. (F) LhS28 staining showed loss of cilia only in the center of the epithelia [hematoxylin and eosin staining for (A and D)]. Immunohistochemistry for $(\mathrm{B}, \mathrm{C}, \mathrm{E}$, and $\mathrm{F})$ [original magnification: $10 \times$ for $(\mathrm{A}, \mathrm{B}, \mathrm{D}$, and $\mathrm{E})$ and $20 \times$ for $(\mathrm{C}$ and $\mathrm{F})]$.

slides, were observed in 7, 6, and 11 cases, respectively. In serous borderline tumor, UES was found in only 1 case, and SS in 3 cases. In serous adenoma, SS were only found in 2 cases, but no UES was found. Frequency of tubal epithelial abnormalities and severity of malignant potential in serous tumors were associated significantly $(P<0.001)$.

In carcinomas, only serous carcinoma had tubal UES in 7 cases (Table 3). SSs were found in serous carcinoma, endometrioid carcinoma, and malignant Brenner tumor. Mucinous carcinoma and clear cell carcinoma did not have any tubal epithelial abnormalities (Table 3). Frequency of tubal epithelial abnormalities and histologic types of ovarian cancer were correlated significantly $(P<0.005)$.

\section{Tubal Epithelial Abnormalities by Diagnostic Algorithm}

Data for tubal epithelial abnormalities by diagnostic algorithm are shown in Table 3 . In serous adenocarcinoma, STIC, STIL, p53 signature, and

TABLE 3. Tubal epithelial abnormalities in serous tumor and in carcinoma

\begin{tabular}{|c|c|c|c|c|c|c|c|c|}
\hline \multirow[b]{2}{*}{ Histologic type } & \multicolumn{3}{|c|}{ H\&E staining } & \multicolumn{4}{|c|}{ Diagnostic algorithm } & \multirow[b]{2}{*}{ Total } \\
\hline & UES & SS & NS & STIC & STIL & p53 signature & Normal/reactive & \\
\hline Serous carcinoma & 7 & 6 & 11 & 5 & 3 & 0 & 16 & 24 \\
\hline Serous borderline tumor & 1 & 3 & 4 & 0 & 3 & 0 & 5 & 8 \\
\hline Serous adenoma & 0 & 2 & $21 \dagger$ & 0 & 1 & 1 & 219 & 23 \\
\hline Total & 8 & 11 & 36 & 5 & 7 & 1 & 42 & 55 \\
\hline Serous carcinoma & 7 & 6 & 11 & 5 & 3 & 0 & 16 & 24 \\
\hline Mucinous carcinoma & 0 & 0 & 14 & 0 & 0 & 1 & 13 & 14 \\
\hline Endometrioid carcinoma & 0 & 3 & 19 & 0 & 0 & 3 & 19 & 22 \\
\hline Clear cell carcinoma & 0 & 0 & 5 & 0 & 0 & 0 & 5 & 5 \\
\hline Malignant Brenner tumor & 0 & 1 & $1 \ddagger$ & 0 & 1 & 0 & 19 & 2 \\
\hline Total & 7 & 10 & 50 & 5 & 4 & 4 & 54 & 67 \\
\hline
\end{tabular}

H\&E indicates hematoxylin and eosin; NS, not suspicious for STIC; SS, suspicious for STIC; STIC, serous tubal intraepithelial carcinoma;

STIL, serous tubal intraepithelial lesion; UES, unequivocal for STIC.

$\dagger P<0.001$.

- $P<0.05$

$\dagger P<0.005$. 
normal/reactive evaluated by diagnostic algorithm, were observed in 5, 3, 0, and 16 cases, respectively. In serous borderline tumor, STILs were found in 3 cases. In serous adenoma, STIL and p53 signature were found in 1 case each. Severity of malignant potential in serous tumors was significantly associated with the frequency of tubal epithelial abnormalities $(P<0.05)$.

In carcinomas, only serous carcinoma had STICs. STILs were only found in fallopian tubes of serous carcinoma and malignant Brenner tumor. p53 signature was found in mucinous carcinoma and in endometrioid carcinoma (Table 3). In different histologic types of ovarian cancer, the frequency of tubal epithelial abnormalities differed significantly $(P<0.05)$.

Mean ages of the patients with STIC, STIL, p53 signature, and normal/reactive were $64(\mathrm{SE} \pm 7.3), 60$ $( \pm 4.8), 60( \pm 3.2)$, and $57( \pm 1.7) \mathrm{yr}$, respectively. Those were not different significantly.

\section{PAX8 Expression in Tubal Epithelia}

In serous adenocarcinoma, PAX8 was positive in $96 \%$ of the cases (Table 4). In serous borderline tumor and serous adenoma, PAX8 was positive in $75 \%$ and $65 \%$, respectively (Table 4 ). Severity of malignant potential in serous tumor was significantly associated with PAX8 positivity $(P<0.05)$. In PAX8positive cases, PAX8 (E) was observed in $78 \%, 67 \%$, and $80 \%$ among cases with serous adenocarcinoma, serous borderline tumor, and serous adenoma, respectively. These were not statistically significant.

In serous, mucinous, endometrioid, clear cell carcinoma, and malignant Brenner tumor, PAX8 was positive in $96 \%, 79 \%, 82 \%, 40 \%$, and $100 \%$, respectively (Table 4). In different histologic types of ovarian cancer, the frequency of PAX8 positivity differed significantly $(P<0.05)$. In PAX8-positive cases, PAX8 (E) was observed in 79\%, 27\%, 28\%, $50 \%$, and $100 \%$ among cases with serous carcinoma, mucinous carcinoma, endometrioid carcinoma, clear cell carcinoma, and malignant Brenner tumor, respectively. Those also differed significantly (Table 4 ) $(P<0.01)$.

Mean age for PAX8-positive patients $(\mathrm{n}=77)$ was $62( \pm 1.4) \mathrm{yr}$, and that for PAX8-negative patients $(\mathrm{n}=21)$ was $42( \pm 3.3)$ yr. PAX8-positive patients are significantly older than PAX8-negative patients $(P<0.0001)$. Among PAX8-positive patients, mean age for the patients with PAX8 (E) expression $(\mathrm{n}=45)$ was $65( \pm 1.7) \mathrm{yr}$, whereas that for the patients with $\operatorname{PAX} 8(\mathrm{H})$ expression $(\mathrm{n}=32)$ was 56 $( \pm 2.1)$ yr. The patients with PAX8 (E) expression were also significantly older than those with PAX8 $(\mathrm{H})$ expression $(P<0.01)$.

\section{DISCUSSION}

Recent advance in molecular genetic studies divides ovarian cancer into 2 categories, such as Type I and Type II tumors (2). Genes related with Type I tumors include $K R A S(19,20), B R A F(19,20), \operatorname{PTEN}(21)$, CTNNBI (22), PIK3CA (23), ARIDIA (24,25), etc. In contrast, most of Type II tumors have p53 mutations $(26,27)$. The dualistic model of ovarian carcinogenesis best applies for serous ovarian cancer (2). In molecular studies, $B R A F$ or $K R A S$ mutations have been reported in about $2 / 3$ of low-grade serous tumors $(19,20)$. In contrast, over $95 \%$ of high-grade serous carcinomas have been reported to have $p 53$ mutations (26).

TABLE 4. PAX8 expression of tubal epithelia in serous tumor and in carcinoma

\begin{tabular}{|c|c|c|c|c|c|c|}
\hline \multirow[b]{2}{*}{ Histologic type } & \multicolumn{2}{|c|}{ PAX8 } & \multirow[b]{2}{*}{ Total } & \multicolumn{2}{|c|}{ Grade of staining } & \multirow[b]{2}{*}{ Total } \\
\hline & Positive & Negative & & PAX8 (E) & PAX8 $(\mathrm{H})$ & \\
\hline Serous carcinoma & 23 & 1 & 24 & 18 & 5 & 23 \\
\hline Serous borderline tumor & 6 & 2 & 8 & 4 & 2 & 6 \\
\hline Serous adenoma & 15 & $8^{*}$ & 23 & 12 & $3 末$ & 15 \\
\hline Total & 44 & 11 & 55 & 34 & 10 & 44 \\
\hline Serous carcinoma & 23 & 1 & 24 & 18 & 5 & 23 \\
\hline Mucinous carcinoma & 11 & 3 & 14 & 3 & 8 & 11 \\
\hline Endometrioid carcinoma & 18 & 4 & 22 & 5 & 13 & 18 \\
\hline Clear cell carcinoma & 2 & 3 & 5 & 1 & 1 & 2 \\
\hline Malignant Brenner tumor & 2 & $0^{*}$ & 2 & 2 & $0 \dagger$ & 2 \\
\hline Total & 56 & 11 & 67 & 29 & 27 & 56 \\
\hline
\end{tabular}

$* P<0.05$.

$\dagger P<0.01$.

$* *$ Not significant. 
Epidemiological studies suggest the relationship of uninterrupted ovulation with a risk of ovarian cancer (28-30). It is evidenced by a number of studies, which indicate that oral contraceptives have a protective effect on ovarian cancer $(28,29,31)$. Ovarian cancer is often advanced when diagnosed. However, clinicopathologic evidence shows that clinical stage distribution differs between histologic types of epithelial ovarian cancer. Especially, serous carcinoma has higher clinical stages than the carcinomas of other histologic types (32), as also shown in our study.

Since the discovery of familial ovarian cancer genes $B R C A 1$ and BRCA2, frequent association of STICs and tubal dysplasia by thorough examination of fallopian tubes taken by prophylactic salpingooophorectomy has been reported (3-13). It should also be noted that a number of studies have documented a frequent association of STICs with serous ovarian cancer irrespective of $B R C A 1$ or $B R C A 2$ mutations $(12,33,34)$. Tang et al. (34) reported that $19 \%$ of serous carcinoma in 300 consecutive gynecologic cases had STICs in their fallopian tubes. Furthermore, Maeda et al. (33) reported that $47 \%$ of serous carcinoma had STICs. They did not find STICs in the fallopian tubes of the ovarian cancer cases with the other histologic types. These lead to the hypothesis, in which STICs are the precursor lesions of the serous ovarian cancer.

Usually STICs are diagnosed on H\&E-stained slides. However, interobserver reproducibility is low because of morphologic complexities in tubal epithelium (14). Recently, Visvanathan et al. (15) reported the use of algorithm for the diagnosis of STIC. Vang et al. (16) showed the algorithm worked well in their validation study.

In this study, we have shown the frequent association of the tubal epithelial abnormalities with serous carcinoma. This association was significant when compared with serous borderline tumor and serous adenoma $(P<0.05$, Table 3$)$. This was also found among different histologic types of epithelial ovarian carcinomas $(P<0.05$, Table 3$)$. As Maeda et al. (33) reported, we found STICs only in the fallopian tubes of the cases with serous carcinoma. Because most of the cases in our study were high grade, frequent association of STICs in serous carcinoma might reflect Type II pathway of ovarian carcinogenesis.

Association of STIC with serous carcinoma was only $20 \%$ in our study. This is comparable with $19 \%$ by the report of Tang et al. (34). However, other reports described the higher association of STICs with serous carcinoma, in a range of $47 \%$ to $75 \%(10,33,35)$. This might be reflected by the lower percentage of tubal sectioning by SEE-FIM protocol (9). Still, significant association of STICs with serous carcinoma in our study supports the hypothesis in which serous ovarian cancer originate from the FTE.

$P A X 8$, one of the human paired box genes $P A X 1$ through $P A X 9$, is a transcription factor that is essential for embryonic development of the kidney, müllerian organs, and thyroid $(17,36)$. Immunohistochemical expression of PAX8 is observed in normal tissues including renal tubular cells, ovarian surface epithelial cells, endocervical epithelial cells, endometrial epithelial cells, seminal vesicle epithelial cells, epididymal epithelial cells, thyroid epithelial cells, lymphoid cells, and pancreatic islet cells, as well as in primary neoplasms from those tissues sited above. In tubal epithelium secretory cells and basal cells, but not ciliated cells, express PAX8 (17). It has been known that secretory cells of tubal epithelial cells proliferate in the patients who have $B R C A$ mutations (13). As Visvanathan et al. (15) reported, morphologic diagnosis of STICs requires 2 or more diagnostic features in 10 or more consecutive secretory cells in the fallopian tube. We applied the same criteria of SCP to evaluate PAX8 expression. $P A X 2$ is also one of the human paired box genes, and closely related with $P A X 8$. Tubal secretory cells express PAX2 as well as PAX8. Laury et al. (18) reported higher frequency of secretory cell outgrowths in the cases with serous borderline tumor by negative expression of PAX2 and p73 immunohistochemically. As differed from PAX2, PAX8 is always expressed in tubal secretory cells. We used PAX8 because it is the positive marker to detect the SCP. The loss of ciliated cells was confirmed by using the ciliated body marker LhS28. This is the first study to investigate the SCP by PAX8 and $\mathrm{LhS} 28$ expression. In this study we observed the SCP more frequently in serous carcinoma than in borderline tumor or serous adenoma $(P<0.05)$. Interestingly, PAX8-positive cases were observed more frequently in serous carcinoma than in carcinoma of the other histology $(P<0.01)$. In addition, all but one case with tubal epithelial abnormalities (i.e. other than normal/ reactive) showed positive PAX8 expression (data not shown). Our observation was concordant with the observation of Laury et al. (18). Although our data does not imply with certainty that the SCP of the fallopian tube would predispose serous carcinoma, 
PAX8-positive SCP might still have a meaningful association with serous carcinoma. It is also intriguing that the cases with PAX8-positive expression are significantly older than those with negative expression $(P<0.0001)$. In addition, the cases with PAX8 (E) expression were significantly older than those with PAX8 $(\mathrm{H})$ expression $(P<0.01)$. These reflect that the number of tubal secretory cells will increase in older ages. The association of SCP with older age is concordant with the prevalence of ovarian cancer in older ages.

When the dualistic model of ovarian carcinogenesis is applied to the tubal epithelium, STICs are thought to be a direct precursor of Type II serous carcinoma. A precursor of Type I tumor, especially low-grade serous carcinoma, is known to be serous borderline tumor. However, the association between tubal epiethelium and serous borderline tumor is still not apparent. As Laury et al. (18) indicated, PAX2-null secretory cell outgrowths might be a precursor for low-grade serous carcinoma. In our study, PAX8-positive SCP was prevalent in borderline serous tumor as well as Type II tumors. So, SCP might be a source for precursor lesions of Type I as well as Type II tumors.

In summary, the presence of tubal epithelial abnormalities was significantly associated with malignant potential among serous tumors. Also STICs are exclusively found in serous carcinoma among epithelial ovarian cancer. Of note, PAX8 expression in fallopian tubes is also associated with malignant potential among serous tumor and more prevalent in serous histology among epithelial ovarian cancer. PAX8 overexpression was also correlated with older age. Our observations are concordant with the recent hypothesis of ovarian carcinogenesis in which serous ovarian cancer originates from the FTE. Further investigation would be necessary to confirm this issue.

ACKNOWLEDGMENTS: The authors thank Dr Steven G. Silverberg for his kind suggestion. They also thank Shinya Sasaki, Miho Takase, Risa Okatake, and Shunsaku Suzuki for their kind technical assistance.

\section{REFERENCES}

1. Ushijima K. Current status of gynecologic cancer in Japan. J Gynecol Oncol 2009;20:67-71.

2. Kurman RJ, Shih LM. Molecular pathogenesis and extraovarian origin of epithelial ovarian cancer. Shifting the paradigm. Hum Pathol 2011;42:918-31.

3. Finch A, Beiner M, Lubinski J, et al. Salgingo-oophorectomy and the risk of ovarian, fallopian tube, and peritoneal cancers in women with a BRCA1 or BRCA2 mutation. JAMA 2006; 296:185-92.
4. Rebbeck TR, Kauff ND, Domchek SM. Meta-analysis of risk reduction estimates associated with risk-reducing salpingooophorectomy in BRCA1 or BRCA2 mutation carriers. $J$ Natl Caner Inst 2009;101:80-7.

5. Finch A, Evans G, Narod SA. BRCA carriers, prophylactic salpingo-oophorectomy and menopause: clinical management considerations and recommendations. Women Health 2012;8: 543-55.

6. Piek JMJ, van Diest PJ, Zweemer RP, et al. Dysplastic changes in prophylactically removed fallopian tubes of women predisposed to developing ovarian cancer. J Pathol 2001;195:451-6.

7. Kauff ND, Satagopan JM, Robson MF, et al. Risk-reducing salpingo-oophorectomy in women with a BRCA1 or BRCA2 mutation. $N$ Eng $J$ Med 2002;346:1609-15.

8. Olopade OI, Artioli G. Efficacy of risk-reducing salpingooophorectomy in women with BRCA-1 and BRCA-2 mutations. Breast $J$ 2004;10:S5-9.

9. Powell CB, Kenley E, Chen LM, et al. Risk-reducing salpingooophorectomy in BRCA mutation carriers: role of serial sectioning in the detection of occult malignancy. J Clin Oncol 2005;23:127-32

10. Finch A, Shaw P, Rosen B, et al. Clinical and pathologic findings of prophylactic salpingo-oophorectomies in 159 BRCA1 and BRCA2 carriers. Gynecol Oncol 2006;100:58-64.

11. Leunen K, Legius E, Moerman P, et al. Prophylactic salpingooophorectomy in 51 women with familial breast-ovarian cancer: importance of fallopian tube dysplasia. Int J Gynecol Cancer 2006;16:183-8.

12. Medeiros F, Muto MG, Lee Y, et al. The tubal fimbria is a preferred site for early adenocarcinoma in women with familial ovarian cancer syndrome. Am J Surg Pathol 2006;30:230-6.

13. Crum CP, Dropkin R, Kindelberger D, et al. Lessons from BRCA: the tubal fimbria emerges as an origin for pelvic serous cancer. Clin Med Res 2007;5:35-44.

14. Carlson JW, Jarboe EA, Kindelberger D, et al. Serous tubal intraepithelial carcinoma: diagnostic reproducibility and its implications. Int J Gynecol Pathol 2010;29:310-4.

15. Visvanathan K, Vang R, Shaw P, et al. Diagnosis of serous tubal intraepithelial carcinoma (STIC) based on morphologic and immunohistochemical features. A reproducibility study. Am J Surg Pathol 2011;35:1766-75.

16. Vang R, Visvanathan K, Gross A, et al. Validation of an algorithm for the diagnosis of serous tubal intraepithelial carcinoma. Int J Gynecol Pathol 2012;31:243-53.

17. Ozcan A, Shen SS, Hamilton C, et al. PAX8 expression in nonneoplastic tissues, primary tumors, and metastatic tumors: a comprehensive immunohistochemical study. Mod Pathol 2011; 24:751-64.

18. Laury AR, Ning G, Quick CM, et al. Fallopian tube correlates of ovarian serous borderline tumors. Am J Surg Pathol 2011; 35:1759-65.

19. Singer G, Oldt R, Cohen Y, et al. Mutations in BRAF and KRAS characterize the development of low-grade ovarian serous carcinoma. J Natl Cancer Inst 2003;95:484-6.

20. Sieben NLG, Macropoulos P, GMJM Roemen, et al. In ovarian neoplasms, BRAF, but not KRAS, mutations are restricted to low-grade serous tumours. J Pathol 2004;202: $336-40$.

21. Obata K, Morland SJ, Watson RH, et al. Frequent PTEN/ MMAC mutations in endometrioid but not serous or mucinous epithelial ovarian tumors. Cancer Res 1998;58: 2095-7.

22. Catasus L, Bussaglia E, Rodrguez I, et al. Molecular genetic alteration in endometrioid carcinomas of the ovary: similar frequency of beta-catenin abnormalities but lower rate of microsatellite instability and PTEN alterations than in uterine endometrioid carcinomas. Hum Pathol 2004;35:1360-8. 
23. Campbell IG, Russell SE, Choong DYH, et al. Mutation of the PIK3CA gene in ovarian and breast cancer. Cancer Res 2004;64:7678-81.

24. Jones S, Wang TL, Shih LM, et al. Frequent mutations of chromatin remodeling gene ARID1A in ovarian clear cell carcinoma. Science 2010;330:228-31.

25. Wiegand KC, Shah SP, Al-Agha OM, et al. ARID1A mutations in endometriosis-associated ovarian carcinomas. $N$ Engl J Med 2010;363:1532-43.

26. Ahmed AA, Etemadmoghadam D, Temple J, et al. Driver mutations in TP53 are ubiquitous in high grade serous carcinoma of the ovary. $J$ Pathol 2010;221:49-56.

27. Kurman RJ, Shih LM. Molecular pathogenesis and extraovarian origin of epithelial ovarian cancer. Shifting the paradigm. Hum Pathol 2011;42:918-31.

28. Casagrande JT, Pike MC, Ross RK, et al. "Incessant ovulation" and ovarian cancer. Lancet 1979;2:170-3.

29. Barber HRK, eds. Ovarian Carcinoma, Etiology, Diagnosis, and Treatment.; 3rd ed New York, Berlin, Heidelberg, London, Paris, Tokyo, Hong Kong, Barcelona, Budapest: SpringerVerlag; 1993.

30. Brewer MA, Johnson K, Follen M, et al. Prevention of ovarian cancer: intraepithelial neoplasia. Clin Cancer Res 2003;9:20-30.
31. Collaborative Group on Epidemiological Studies of Ovarian Cancer. Ovarian cancer and oral contraceptives: collaborative reanalysis of data from 45 epidemiological studies including 23257 women with ovarian cancer and 873030 controls. Lancet 2008:371:303-14.

32. Seidman JD, Horkayne-Szakaly I, Haiba M, et al. The histologic type and stage distribution of ovarian carcinomas of surface epithelial origin. Int J Gynecol Pathol 2003;23:41-4.

33. Maeda D, Ota S, Takazawa Y, et al. Mucosal carcinoma of the fallopian tube coexists with ovarian cancer of serous subtype only: a study of Japanese cases. Vichows Arch 2010;457: 597-608.

34. Tang S, Onuma K, Deb P, et al. Frequency of serous tubal intraepithelial carcinoma in various gynecologic malignancies: a study of 300 consecutive cases. Int J Gynecol Pathol 2012; 31:103-10.

35. Kindelberger DW, Lee Y, Miron A, et al. Intraepithelial carcinoma of the fimbria and pelvic serous carcinoma: evidence for a causal relationship. Am J Surg Pathol 2007;31:161-9.

36. Poleev A, Fickensher H, Mundlos S, et al. PAX8, a human paired box gene: isolation and expression in developing thyroid, kidney and Wilm's tumors. Development 1992;116: 611-23. 